\section{Hue discrimination as a function of stimulus luminance*}

\author{
MICHAEL H. SIEGEL and ANNE B. SIEGEL \\ Albion College, Albion, Michigan 49224
}

The relation between hue discrimination and stimulus luminance was investigated. It was found that discrimination was best at the highest luminance and deteriorated at the lowest, except in the yellow region.

One of the classical psychophysical experiments involves determining Os' ability to detect differences between two stimuli which differ in wavelength as a function of the wavelength of the standard stimulus. Studies of this sort have, at various times, been called wavelength discrimination or hue discrimination. Because wavelength is a physical rather than a psychological property of the stimulus, the present authors feel that "wavelength discrimination" is inappropriate. This kind of study will be referred to as hue discrimination throughout the remainder of this paper despite the fact that, strictly speaking, both the hue and the saturation of the stimulus is changed when spectral wavelength is changed.

Research in vision over the last century provides many examples of attempts to measure hue discrimination. The study by L. A. Jones (1917) in the first issue of the Journal of the Optical Society of America is still frequently cited as an example of the relationship of delta lambda to lambda, sensitivity to wavelength differences as a function of wavelength. Later, others extended the classical studies. Much of the work prior to 1930 has been summarized by Judd (1932).

By the early 1960 s, research was still active in this area. Results of hue discrimination studies have been reported by Weale (1953), McCree (1960), Siegel (1964), Bedford and Wyszecki (1958), and Pokorny and Smith (1970) to name just a few. The relation to opponent color theory has been noted by the Hurviches (Hurvich \& Jameson, 1955). Probably the most universally accepted finding among all researchers was that ability to discriminate between two stimuli which differed in wavelength was indeed a function of the wavelength of the standard. Although different studies had important differences about the shape of the hue

* Results of this experiment were reported at the 1970 meeting of the Eastern Psychological Association. The research was supported in part by Public Health Service Grant MH-14281. discrimination curve and about the possible extent of human discrimination ability, it became clear that detecting differences between two stimuli differing in wavelength was best in the regions of the spectrum generally called blue and yellow and worst at the ends of the spectrum and in the green.

Some important questions still remained. One concerned the possible presence of an additional minimum point in the delta lambda curve at very short wavelengths. A second involved the relationship between stimulus luminance and the delta lambda curve.

With respect to the first of these questions, secondary minima in the violet region had been reported in some of the classical literature. One problem with early research, though, was an inability to provide sufficient energy at short wavelengths. Many of the early attempts, in fact, did not have a constant luminance across the spectrum. Of course, this makes interpretation of data involving subtle differences almost meaningless if hue discrimination also depends upon luminance level.

The pioneering work of Purdy in the 1930s demonstrated that color appearance was influenced by the intensity of the stimulus. Other research, both earlier and later than Purdy's, suggested that detecting hue differences was also influenced by stimulus intensity, but no exact relation has been worked out. Connors (1964) found that when stimulus luminance was sufficiently altered, hue discrimination was influenced at $550 \mathrm{~nm}$. Thomson and Trezona (1951) suggest that, as luminance is reduced, discrimination deteriorates in the same proportion for all wavelengths between 490 and $620 \mathrm{~nm}$. Hurvich and Jameson (1961) have found that hue discrimination is affected not only by the stimulus luminance, but also by the hue and the saturation of the preexposure and the surround field. The Hurvich-Jameson data are characterized by less prominent minima at brighter stimulus values. Verriest et al (1963) supported the earlier contention (e.g., Brown, 1951) that hue discrimination is especially affected in the blue region. This is the well-known "tritanopia" effect.

The principal goals of the present study are (1) the further investigation of the shape of the hue discrimination curve, especially as short wavelengths, and ( 2) the study of hue discrimination as influenced by luminance. Additional questions are the extent to which the so-called invariant hues remain impervious to the effect of luminance differences and the verification of tritanopia at reduced luminance levels.

\section{APPARATUS}

A schematic drawing of the viewing situation is provided as Fig. 1. Two Bausch and Lomb grating monochromators, each with a $500-\mathrm{mm}$ focal length, were used to provide a circular, vertically divided field which subtended $3 \mathrm{deg}$ at the $O$ 's eye. An optical system placed both exit slits in Maxwellian view. One instrument supplied the standard and the other the variable half of the stimulus. A

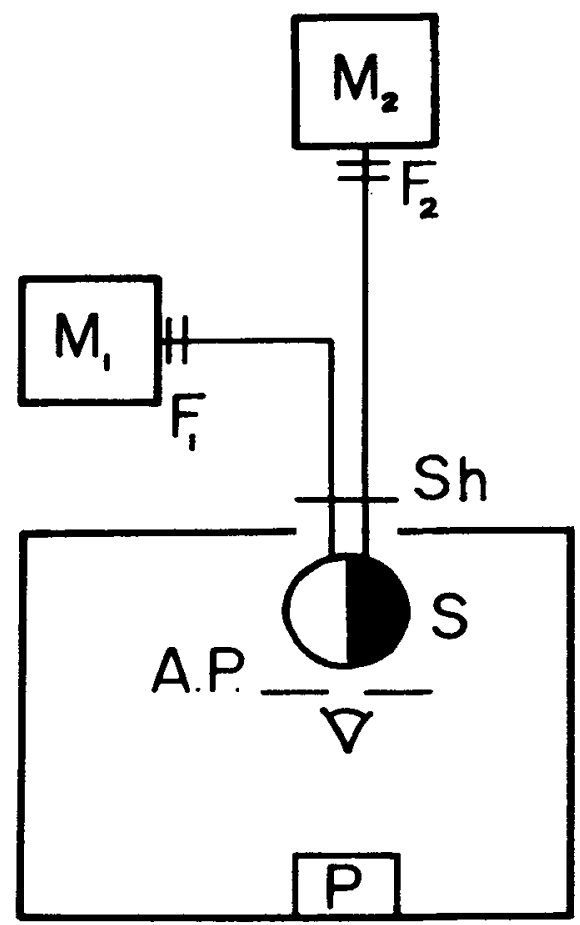

Fig. 1. Schematic drawing of the viewing situation. Light from the monochromators $\left(M_{1}\right.$ and $\left.M_{2}\right)$ are appropriately filtered at $F_{1}$ and $F_{2}$. The optical system provides a Maxwellian view to the observer $(0)$, who views the stimulus (S) through an artificial pupil (A.P.). The .2-sec stimulus exposure is achieved by action of the shutter (Sh). The area surrounding the stimulus is illuminated by a projector (P) with its own light attenuation system. 


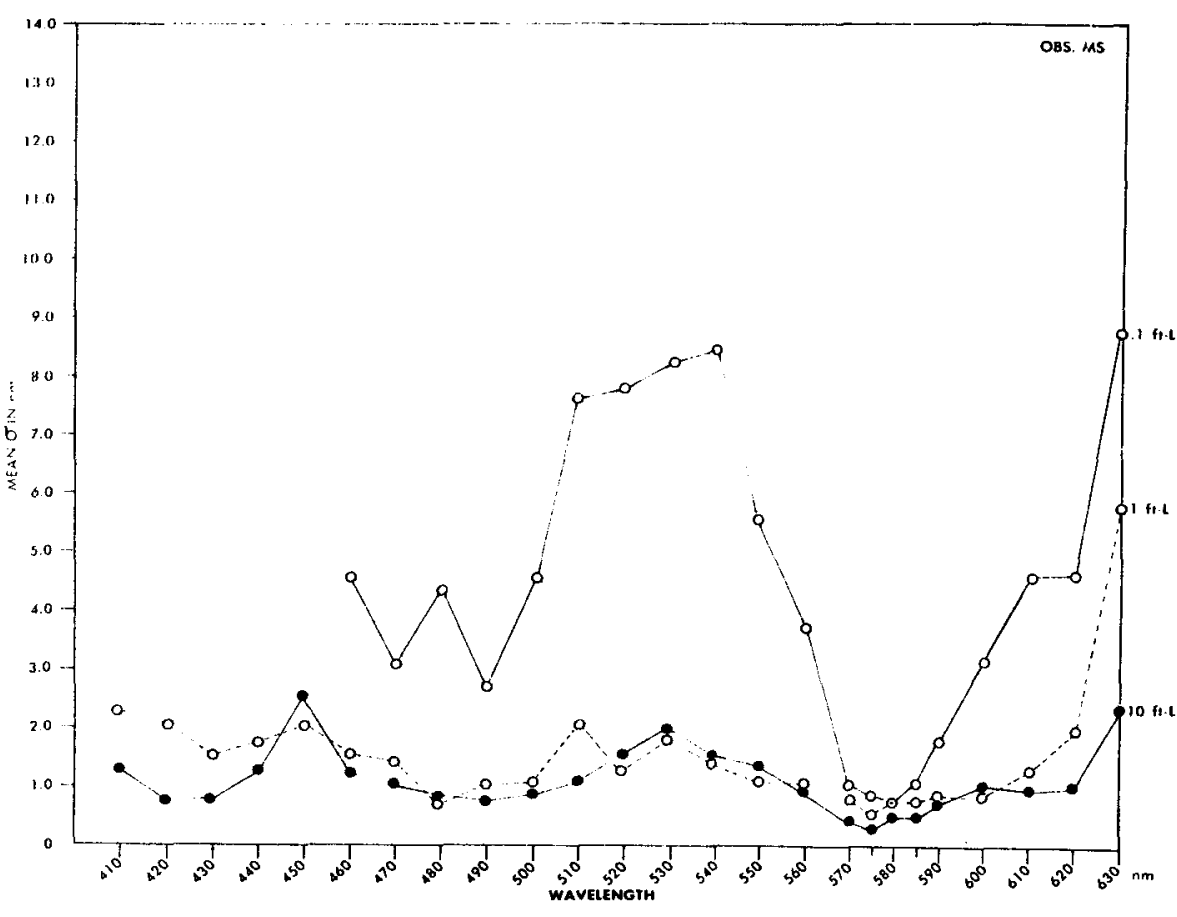

Fig. 2. Hue discrimination for $O$ M.S. at three brightness levels.

dual system of neutral density filters allowed the brightness of the stimulus to be varied and a sector shutter permitted discrete .2-sec exposures. The stimulus appeared through a circular hole in a white cardboard screen, which was illuminated from above and behind the $O$. The color temperature of the surround, which filled the $O$ 's entire visual field, approximated that of Illuminant $C$, and its luminance was maintained at 1 fL throughout the entire experiment. The $O$ sat in a separate booth and viewed the stimulus through a $3-\mathrm{mm}$ artificial pupil with his right eye. The left eye was occluded. A head- and chinrest was used to minimize head movement.

Calibrations of wavelength, exposure duration, and stimulus brightness both before and after experimentation indicated no changes. A psychophysical calibration of the stimulus brightness was made necessary by the use of a Maxwellian view optical system. The luminance of the surround was set in turn to 0.1 , 1.0 , and $10 \mathrm{fL}$, using a calibrated photometer, a Spectra Brightness Spot Meter, Model U B $11 / 2$. Because the surround illumination was from above, there was a slight luminance gradient. The field appeared uniformly bright to the Os. All photometric measurements were made on a 1/2-in.-diam spot immediately to the left of the aperture through which the stimulus appeared. The standard (left) half of the stimulus field at $570 \mathrm{~nm}$ was matched in brightness to the surround. Next, heterochromatic brightness matches were made between the halves of the field for each of the 25 wavelengths studied.

\section{OBSERVERS}

There were three Os. All were emmetropic and color normal, as determined by the Farnsworth Munsell 100 Hue Test and the American Optical Company Pseudo Isochromatic plates. Both of the authors were highly practiced, and the third $O$ had had several weeks of intensive training in the judgments required. Only the right eye was used.

\section{PROCEDURE}

The method of constant stimulus differences was used. In any given experimental session, the $O$ was asked to discriminate between the hue of the standard stimulus, whose wavelength remained invariant for the entire session, and the hue of the comparison stimulus. In one session, the five cumparison stimuli were chosen to be equal to or longer in wavelength than the standard stimulus, and the $O$ was required to respond yes or no to a question such as, "Is the stimulus on the right redder than the stimulus on the left?" In the next session, the same standard stimulus would be compared with five other comparison stimuli whose wavelengths were equal to or shorter than that of the standard. Now the $O$ was required to respond yes or no to a question such as, "Is the stimulus on the right yellower than the stimulus on the left?" In each experimental session, five different comparison stimulus values were presented 10 times each in a random

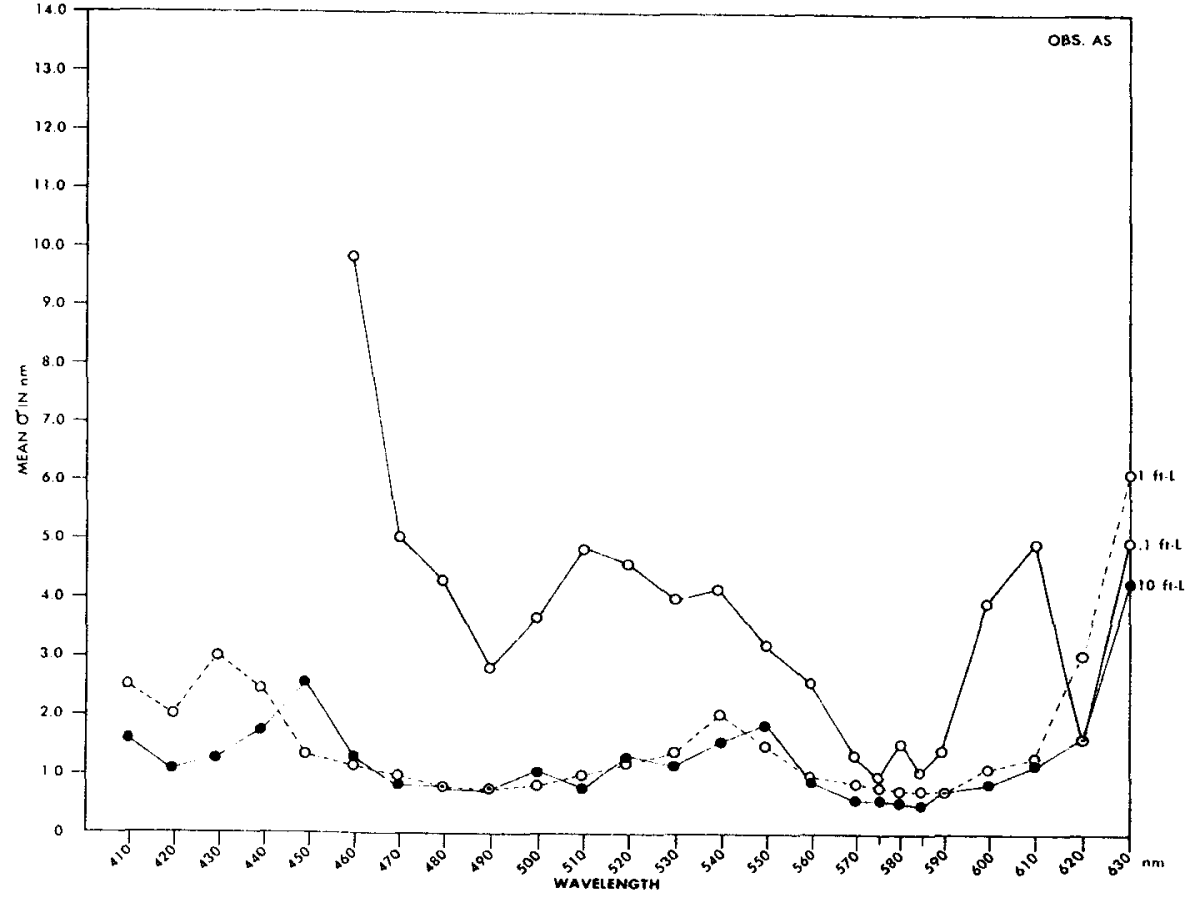

Fig. 3. Hue discrimination for $\mathbf{O}$ A.S. at three brightness levels. 
order. Data from both sessions were used to calculate results. A day's run consisted of two such sessions. To ensure an adequate sampling, the sessions were rerun so that each data point for each standard stimulus is based upon four separate sessions. The step size for the comparison stimuli was determined in a pretest and varied depending upon the wavelength location of the standard. Extreme values of the comparison stimuli were chosen to obtain a psychometric curve which would be distributed from about $10 \%$ to about $90 \%$ yes responses (Boring, 1917).

This procedure was used for 25 different standard stimulus wavelength values ranging from 410 through $630 \mathrm{~nm}$. Each of these wavelengths was presented at three different brightness levels, corresponding to matches to the surround for surround luminances of $0.1,1.0$, and $10 \mathrm{fL}$. Thus, the experimental data are based upon a total of 300 sessions for each of the three Os: standard stimuli of 25 different wavelengths, at three different brightness values, at each of which comparison stimuli are chosen twice in each of two wavelength directions from the standard. The total experiment, then, is based upon some 45,000 individual judgments.

\section{RESULTS AND DISCUSSION}

The measure used to estimate sensitivity was the standard deviation. When the percentage of "yes"

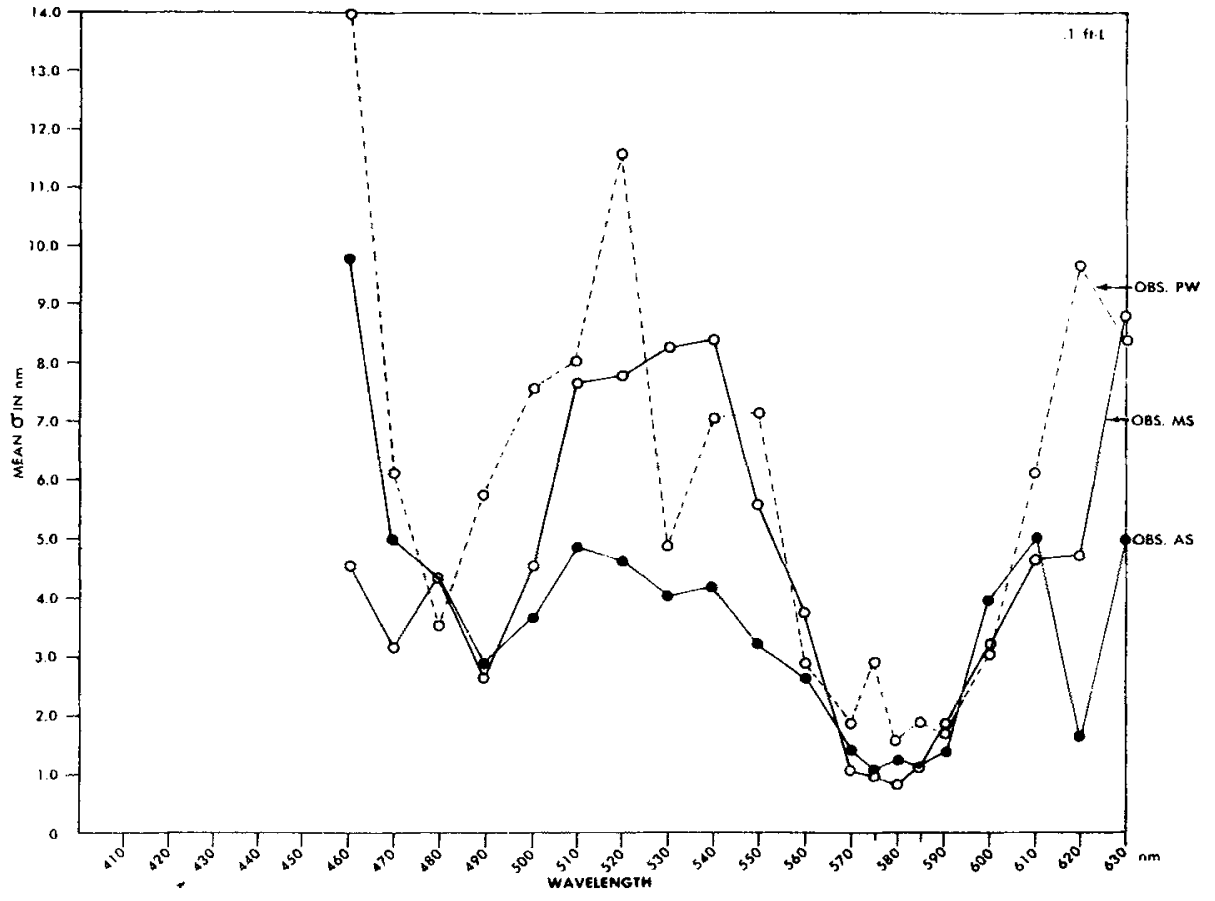

Fig. 5. Hue discrimination at $0.1 \mathrm{fL}$.

responses are plotted against wavelength, the distribution assumes an ogival form. By plotting the data on normal probability paper, it becomes simple to calculate a mean, the wavelength at the $50 \%$ point, and a standard deviation, the difference

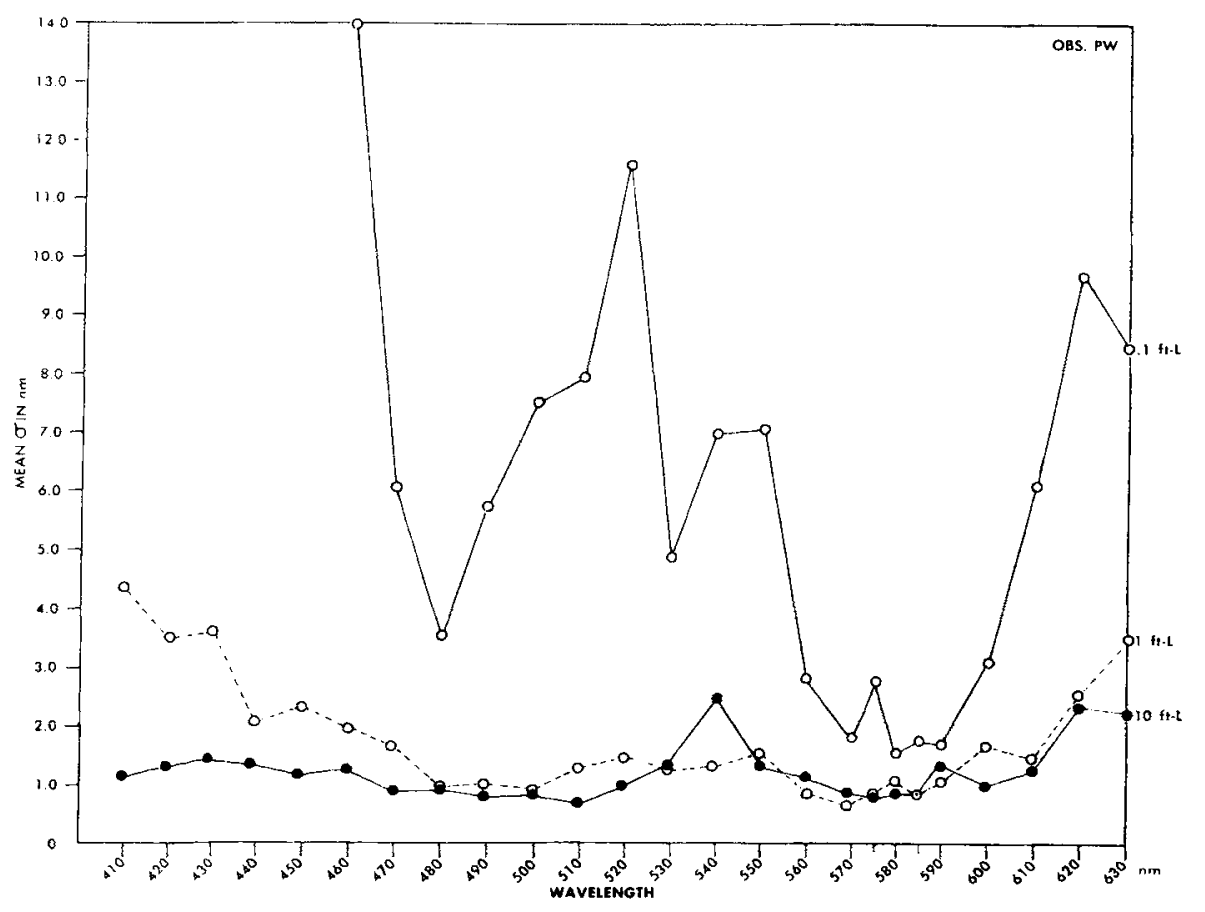

Fig. 4. Hue discrimination for O P.W. at three brightness levels. between the wavelengths corresponding to the $84.13 \%$ and the $50 \%$ points. In an earlier paper, Siegel (1969a) has argued that the standard deviation should be used instead of the more traditional measure, the difference limen or jnd. The standard deviations reported are the arithmetic mean of the four standard deviations, two in each wavelength direction, for a given combination of standard wavelength and brightness level.

Figures 2, 3, and 4 plot the data separately for each $O$ at each of the three stimulus brightness values. The mean standard deviation in $\mathrm{nm}$ appears on the ordinate and wavelength on the abscissa. All Os show greatest sensitivity at the two highest stimulus brightness levels and vastly deteriorated performance at the dimmest level. These results agree with an earlier paper (Siegel, 1969b), which found this effect even at $570 \mathrm{~nm}$, a wavelength at which, in the present study, sensitivity is relatively independent of brightness.

Comparisons among Os are facilitated by replotting the data to show results for all three Os at any given brightness. Figures 5,6 , and 7 do this. Again, mean standard deviation is on the ordinate and wavelength on the abscissa. At both 10 and $1 \mathrm{fL}$, all $\mathrm{Os}$ show distinct minima, or areas of greatest sensitivity to hue differences, around $480-500 \mathrm{~nm}$ in the blue-green region of the spectrum and at about 570-585 $\mathrm{nm}$ in the yellow region. Curves for the two highly trained Os 


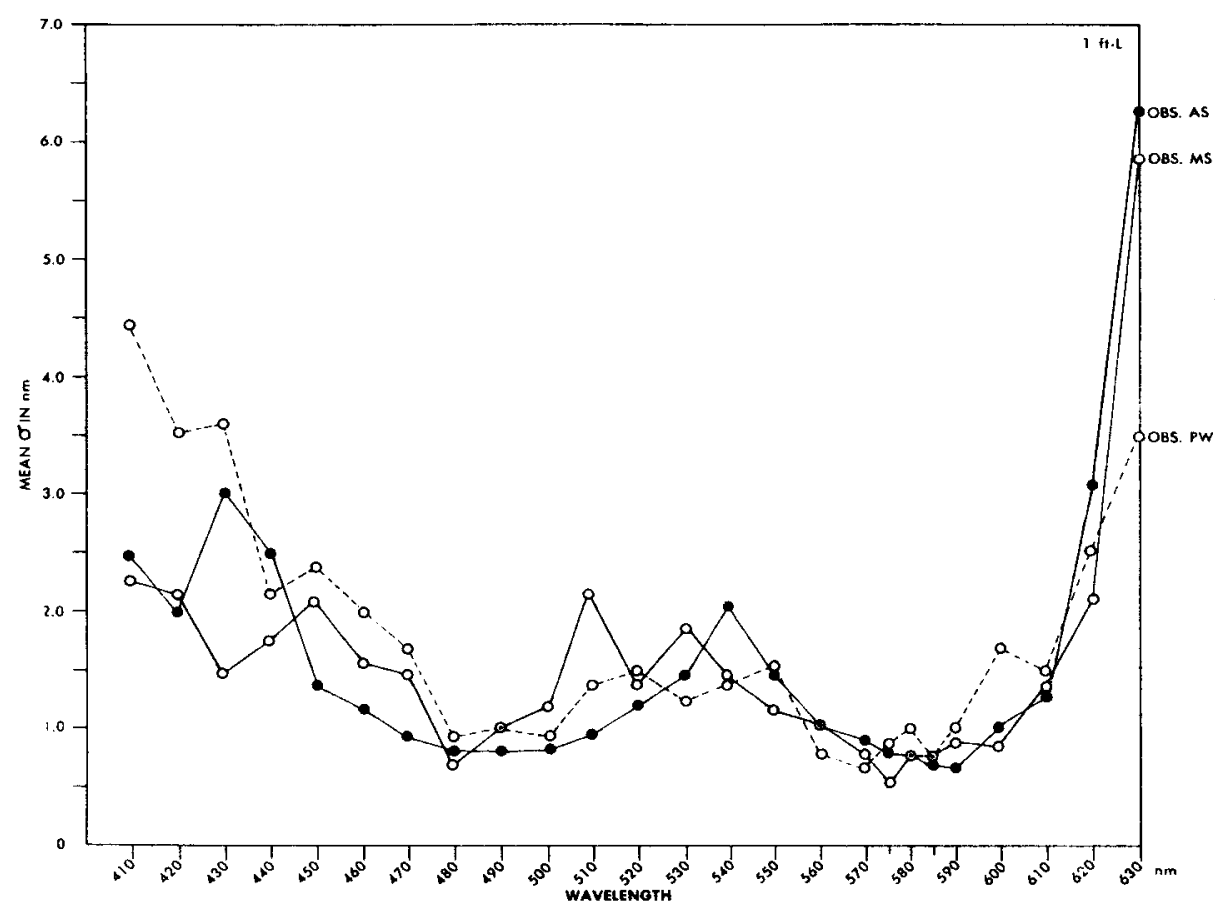

Fig. 6. Hue discrimination at $1.0 \mathrm{fL}$.

also show a third minimum at $420-430 \mathrm{~nm}$ in the violet region for both these brightness levels. All three Os show nearly the same areas of least sensitivity at both 10- and 1 -fL levels. One such area appears at the extreme red end of the spectrum and a second may be found at $530-550 \mathrm{~nm}$ in the yellow-green area.

As brightness was decreased from 10 to $1 \mathrm{fL}$, the areas of greatest and least sensitivity show more variation among Os. When stimulus brightness was further decreased to $0.1 \mathrm{fL}$, even greater individual differences appear, in addition to the generally deteriorated sensitivity already mentioned. Indeed, curves for the three Os seem to approach convergence at only two distinct minima, one at $480-490 \mathrm{~nm}$ in the blue area and the other at about $575-580 \mathrm{~nm}$ in the yellow region. It should also be noted that this yellow area is the only one which seems virtually immune to the effects of stimulus brightness; sensitivity is almost as good at .1 fL as at $10 \mathrm{fL}$.

At a stimulus luminance of $0.1 \mathrm{fL}$, despite the inter- $O$ variability, a clear pattern of reduced sensitivity can be found for all three Os from about 510 to $540 \mathrm{~nm}$. Below $460 \mathrm{~nm}$, discrimination deteriorated so much that meaningful measurement was impossible, although it was attempted. This seems to be a verification of the tritanopic effect at reduced brightness and certainly is at odds with Thomson and Trezona (1951), who failed to
(1) sensitivity decreases greatly as stimulus brightness is decreased to $0.1 \mathrm{fL} ;(2)$ the regions of greatest and least sensitivity are most evident at the highest stimulus brightness; (3) sensitivity to hue differences is almost unaffected by changes in stimulus brightness in the yellow region of the spectrum; (4) the short wavelength portion of the spectrum is most affected by reduced brightness.

To summarize, then, our experiment has substantiated that the two most profound minima in the hue discrimination curve appear at the blue and yellow spectral regions. We have found support for an additional minimum in the violet region, at least with stimuli of high brightness with trained Os. We have found that, aithough discrimination may deteriorate progressively as the stimulus brightness is decreased, no substantial effects of brightness were revealed above $0.1 \mathrm{fL}$. A more complete specification of the influence of brightness on hue discrimination would require the study of values between 0.1 and $1.0 \mathrm{fL}$, since data for these limiting values differed so radically.

Finally, we have found only limited support for the notion that there is a relation between the invariant hues, whose color appearance presumably is not influenced by brightness changes, and hue discrimination ability. Only in the yellow wavelength region does discrimination seem not to be by averaging data from all three it becomes immediately clear that discover this effect. Os also reported difficulty in judging extremely long wavelengths, and discrimination declined correspondingly.

If differences among Os are erased

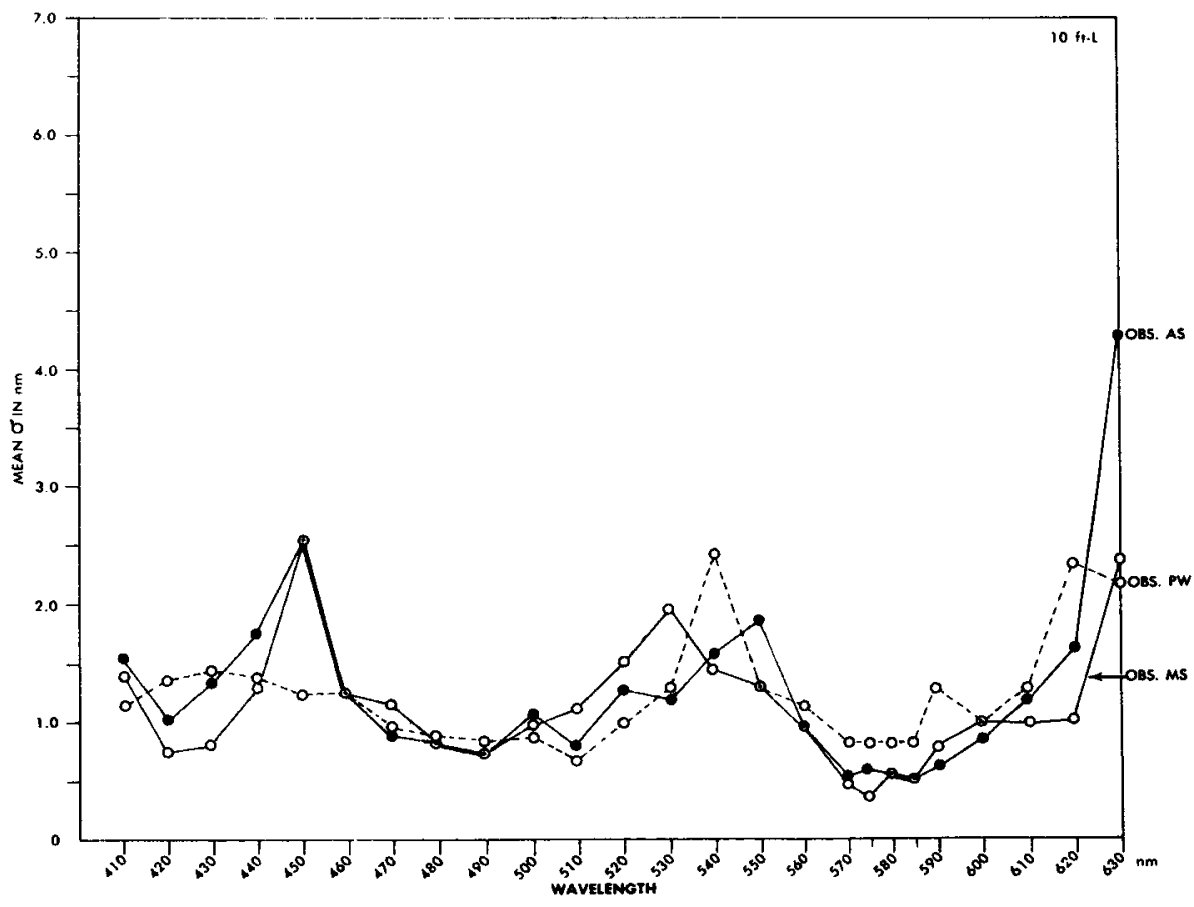

Fig. 7. Hue discrimination at $10 \mathrm{fL}$. 
influenced by brightness changes.

\section{REFERENCES}

BEDFORD, R. E., \& WYSZECKI, G. W W avelength discrimination for point sources. Journal of the Optical Society of America, 1958, 48, $129-135$.

BORING, E. G. Urban's tables and the method of constant stimuli. American Journal of Psychology, 1917, 28 . 280-293.

BROWN $W$ R J The influence of luminance level on visual sensitivity to color differences. Journal of the Optical Society of America, 1951, 41, 684-688.

CONNORS, M. M. Effect of surround and stimulus luminance on the discrimination of hue. Journal of the Optical Society of America, 1964, 54, 693-695.

HURVICH, L. M., \& JAMESON, D. Some quantitative aspects of an opponent-colors theory. II. Brightness, saturation, and hue in normal and dichromatic vision. Journal of the Optical Society of America, 1955, 45, 602-616.
HURVICH, L. M., \& JAMESON, D. H Opponent chromatic induction and wavelength discrimination from the visual wavelength discrimination from the visual
system: Neurophy siology and psychophysics. Freiburg symposium Springer-Verlag, Berlin, 1961, 144-152.

JONES, L. A. The fundamental scale of pure hue and retinal sensibility to hue differences. Journal of the Optical Society of America, 1917, 1, 63-77.

JUDD, D. B. Chromaticity sensibility to stimulus differences. Journal of the Optical Society of America, 1932, 22 $72-108$.

MCCREE, K. J. Small-field tritanopia and the effects of voluntary fixation. Optica Acta, 1960, 7, 317-323.

POKORNY, $j$ \& SITH, V.C. Wavelength discrimination in the presence of added chromatic fields. Journal of the Optical Society of America, 1970, 69, 562-569.

SIEGEL, M, H Discrimination of color. IV. Sensitivity as a function of spectral wavelength, 410 through $500 \mathrm{~nm}$. Journal of the Optical Society of America, 1964,
$54,821-823$.

SIEGEL, M. H. A note on psychophysical measures. Behavior Research Methods \& Instrumentation, 1969a, 1, 289-290.

SIEGEL, M. H. Color discrimination and luminance. Perception \& Psychophysics, $1969 \mathrm{~b}, 6,163-165$.

THOMSON, L. C., \& TREZONA, P. W. The variations of hue discrimination with change in luminance level. Journal of Physiology, 1951, 114, 98-106.

VERRIEST, G. BUYSSENS, A. \& VANDERDONCK, R. Etude quantitative de l'effet qu'exerce sur les resultats de chromatique une diminution non selective due niveau d'un eclairage $C$. Revue d'optique théorique et instrumentale, 1963, 42, 105-119.

WEALE, R. A. Spectral sensitivity and wave-length discrimination of the peripheral retina. Journal of Physiology, 1953, 119, 170-190.

(Accepted for publication June 15, 1972.) 